\title{
In vitro and in vivo Assay for Assessment of the Biological Control Potential of Streptomyces sp. KT
}

\author{
Tatsuya Ohike ${ }^{1}$, Minori Maeda ${ }^{1}$, Tetsuya Matsukawa ${ }^{1}$, Masahiro Okanami ${ }^{1}$, Shin'ichiro Kajiyama ${ }^{1} \&$ Takashi \\ Ano ${ }^{1}$ \\ ${ }^{1}$ Graduate School of Biology-Oriented Science and Technology, Kindai University, Japan \\ Correspondence: Tatsuya Ohike, Graduate School of Biology-Oriented Science and Technology, Kindai \\ University, Japan. E-mail: 1544710254e@waka.kindai.ac.jp
}

Received: November 21, 2017 Accepted: December 8, 2017 Online Published: December 19, 2017

doi:10.5539/jps.v7n1p10

URL: https://doi.org/10.5539/jps.v7n1p10

\begin{abstract}
Rhizoctonia solani is fungal plant pathogen that infects many different host plants. Recently, biological control agents that are friendly to the environment and ecosystems have attracted much attention as an alternative to the use of chemical fungicide which have been used worldwide to control soil borne pathogens including $R$. solani. In this study, 53 strains of actinomycetes isolated from environmental soils, and antifungal activities of them were assessed by the dual culture assay. Strain KT showed strong inhibitory activities against 8 phytopathogenic fungi. A great suppressive effect on $R$. solani growth was observed in the inoculation test of plants using cucumber and chin-geng-sai. In addition, infection of Bipolaris oryzae also could be suppressed in the detached leaf assay using oats. As a result of genetic analysis, it was shown that KT was a species closely related to Streptomyces lavenduligriseus NRRL B- $3173^{\mathrm{T}}$. However, as far as we know, there is no report for biological control agents using $S$. lavenduligriseus. This study suggests that the strain KT may useful as biological control agents to suppress various crop diseases.
\end{abstract}

Keywords: biological control, actinomycetes, plant pathogens

\section{Introduction}

Currently the world population is increasing more than 7 billion people and is projected to reach over 9.8 billion people by 2050 (United Nations, 2017). On the other hand, the arable land area per capita in the world continues to decrease, and it is predicted that in 2050 it will decrease to 30\% compared to 1950 (FAO, 2012). Therefore, improvements of the crop yields must be necessary in limited agricultural land. In order to contribute to solve this problems, reducing the loss of crops in the cultivation steps is important. Plant pathogens are one of the main causes of losses of crop production and pesticides are an effective means to prevent losses in crop production.

Rhizoctonia solani is a very influential plant pathogen in crop production and infects many different host plants, causing damping-off, sheath blight, stem canker, and seed decay. As the disease of Rhizoctonia occurs in all parts of the world, many researches have been done since the discovery of $R$. solani in 1858 (Ogoshi, 1987). In case of rice, yield losses due to sheath blight disease caused by this pathogen was estimated to range from 5 to $10 \%$ in Asia. Moreover, the estimated yield losses increased up to $50 \%$ when susceptible cultivars were grown under suitable conditions for this pathogen. (Lee \& Rush, 1983, Marchetti \& Bollich, 1991, Savary, Willocquet, Elazegui, Castilla, \& Teng, 2000). In potato, $R$. solani causes stem canker in some case, and marketable yield losses have been reported to be approaching 30\% (Banville, 1989). In the case of cucumber fields, the average annual loss in the United States is approximately 7-9\%, which equals to a loss of approximately 4-5 million dollars (Lewis \& Papavizas, 1980). In recent years, up to $75 \%$ mortality in cucumber seedlings in the most affected greenhouse has been reported in countries where greenhouse cultivation is major agriculture (Al-Sa'di, Drenth, Deadman, De Cock, and Aitken, 2007). Therefore, cucumber damping-off is a serious problem not only in agricultural field but also in greenhouse cultivation (Al-Sa'di et al, 2007, Lewis \& Lumsden, 2001). In Japan, it has been reported that $R$. solani $\mathrm{K} 1$ isolated in Kanagawa prefecture in 1986 causes stem rot in various crops such as rice, eggplant, cucumber, spinach, tomato (Asaka \& Shoda, 1996). From these facts, controlling plant pathogens, especially controlling $R$. solani is an important issue for improving agricultural production.

Chemical fungicides have been used worldwide to control soil borne pathogens including $R$. solani (Parry, 1990). However, chemical fungicide application has some problems, such as environmental pollution, deteriorating 
human health, and development of drug-resistant pathogens/insects (Clevo \& Clem, 2001). Furthermore, current chemical agents are not completely effective and Rhizoctonia disease remains to be a persistent problem. Therefore, biological control agents that are friendly to the environment and ecosystems have attracted much attention as an alternative to the use of chemical fungicide.

In recent years, biocontrol of soil born disease, particularly using antibiotic activity of actinomycetes, has been put forward as an alternative to chemical control agents. Actinomycetes are filamentous soil bacteria and also known for their ability to produce many antibacterial/antifungal compounds (Sanglier, Haag, Huck and Fehr, 1996, Jiayue et al., 2016). They also secrete extracellular hydrolytic enzymes such as chitinases and glucanases, which can degrade components in the cell walls of plant pathogens (Castillo et al., 2016, Sakdapetsiri et al., 2016). Therefore, actinomycetes are promising group of root-colonizing microbes where they may influence plants by promoting growth and protecting them from diseases. However, there have been a few reports under unsterilized condition, a similar condition at open field. The objective of this study is to isolate an actinomycete that has a strong antifungal activity and bring out their potential ability to suppress the diseases caused by $R$. solani and other phytopathogens.

\section{Method}

\subsection{Microorganisms}

Actinomycete strain KT was isolated from the soil sample collected in Wakayama, Japan and incubated on PDA (PDA; BD Difco) at $24{ }^{\circ} \mathrm{C}$ for 7 days. Isolated colonies of the actinomycetes were preserved in glycerol suspension $(15 \% \mathrm{v} / \mathrm{v})$ as a stock at $-30{ }^{\circ} \mathrm{C}$. The plant pathogenic fungi [(Bipolaris oryzae MAFF 305382, Colletotrichum echinochloae MAFF 305460, Colletotrichum orbiculare MAFF 306685, Fusarium oxysporum MAFF 103038, Fusarium solani MAFF 235170, Monilinia fructigena MAFF 305640, Phytophthora infestans MAFF 236324, Rhizoctonia solani MAFF 235846), Rhizoctonia solani K-1] were incubated on potato dextrose agar (PDA) at $24^{\circ} \mathrm{C}$ for 7 days before use.

\subsection{Identification of Actinomycete Strain KT}

Phylogenetic analysis based on 16S rDNA sequence and morphological observation were done as follows. Bacterial 16S rRNA genes were PCR-amplified with primers 9F (5'-GAGTTTGATCCTGGCTCAG) and 1510R (5'-GGCTACCTTGTTACGA). The 16S rRNA gene sequences determined were compared with those retrieved from DNA database of APORON DB-BA 11.0 (Techno Suruga Lab., Shizuoka, Japan) and GenBank/EMBL/DDBJ using a BLAST homology search, and phylogenetic tree was constructed to ascertain the phylogenetic position of the actinomycete strain KT. Phylogenetic trees were generated by the neighbor-joining method. Gene sequencing and phylogenetic analysis were carried out at Techno Suruga Lab., Co., Ltd. (Shizuoka, Japan).

\subsection{Dual Culture Assay for in vitro Inhibition of Mycelial Growth of Plant Pathogens}

Antifungal activity of KT against plant pathogens was evaluated by using dual assay. An agar plug of $0.7-\mathrm{cm}$ diameter from actively growing fungal mycelia was placed on the perimeters of the PDA plate at a distance of $1.5 \mathrm{~cm}$, and then strain KT was inoculated on the opposite of the fungal mycelia. Plates were incubated for 7-14 days at $24^{\circ} \mathrm{C}$. The zone of inhibition was measured as halo area around the actinomycete strain KT, and inhibition index was determined by the following criteria; $+:<20 \mathrm{~cm}^{2},++: 20-30 \mathrm{~cm}^{2},+++:>30 \mathrm{~cm}^{2}$.

\subsection{Infection Control of Isolated Actinomycete Strain KT against R. solani}

Cucumis sativus L. (cucumber) and Brassica rapa var. chinensis (qing-geng-cai) were used for infection control test. Seeds were soaked in $70 \%$ ethanol for $10 \mathrm{sec}$, and sterilized with $1 \% \mathrm{NaClO}$ for $10 \mathrm{~min}$, finally rinsed three times with sterile distilled water. Sterilized seeds were germinated under dark conditions for 3 days on water agar plate. The germinated seeds were then transferred to agripot containing modified Hyponex medium (Hyponex powder; $0.0375 \%$, sucrose; $0.35 \%$, agar; $0.8 \%$, pH6.0), with 3 (cucumber) or 5 (qing-geng-cai) seeds in one pot. KT was cultured in PDB medium at $24^{\circ} \mathrm{C}, 1500 \mathrm{rpm}$ (stirred culture) for 3 days and $200 \mu \mathrm{L}$ of the culture broth was spread in the agripot on the day of transplant. Five days after the transplant, the infection assay was carried out by placing $0.7-\mathrm{cm}$-diameter agar plugs of plant pathogens onto the center of agripots. Biocontrol effect was assessed after the plants were grown in growth chamber for $15-20$ days at $28^{\circ} \mathrm{C}, 12 \mathrm{~h}$ light/dark condition.

Seed and soil were not sterilized in the soil experiment, and $2 \mathrm{~g}$ of wet hyphae of KT were used in the experiment. Disease severity was evaluated according to the $0-5$ indexes ( 0 : no disease, 1 : browning of root, 2 : browning of stem, 3: wilting of plants, 4: lodging of plants, 5: plant death), and determined by the following equation; 


$$
\text { Disease index }=\frac{\sum(\text { score of disease } * \text { number of plants })}{\text { total number of plants }}
$$

\subsection{Detached Leaf Assay of Isolated Actinomycete Strain KT against B. oryzae}

Avena sativa (oat) was used for detached leaf assay. Plants at 2 to 3 true leaf stage in pots were harvested and washed with distilled water. They were cut into uniform lengths of $1.5 \mathrm{~cm}$ and cut edge was sealed with paraffin. Sealed leaves were sterilized with $1 \% \mathrm{NaClO}$ for $90 \mathrm{sec}$ and rinsed three times with sterile distilled water. After sterilized, leaves were soaked in KT culture for $10 \mathrm{~min}$. Then, leaves were placed in 12-well microplate with sterilized moistened gauze. For the detached leaf assay, mycelial plug $\left(4 \mathrm{~mm}^{2}\right)$ of Bipolaris oryzae from PDA medium was inoculated onto the upper surface of the Avena leaves pierced with needle of syringe followed by the incubation at $25^{\circ} \mathrm{C}, 12$-h light/dark conditions for 12 days. Disease severity was evaluated according to the leaf area covered by the fungus by the following 0-4 indexes (0: no disease, 1 : hyphal formation around the agar pieces, 2: hyphal coverage of less than 50\%, 3: hyphal coverage of 50-80\%, 4: completely covered with hyphe).

\section{Results}

\subsection{Dual Culture Assay for in Vitro Inhibition of Mycelial Growth of Plant Pathogens}

Fifty-three actinomycete strains were isolated from environmental soil samples, and anti-fungal activities of them were assessed by the dual culture assay using R. solani. Among them, an isolate named KT showed strong suppressive effects on the growth of $R$. solani. The inhibitory effect for other phytopathogens was assessed in the same way as $R$. solani. Antifungal activity of the strain KT was assessed by measuring the inhibition zones present after incubation of dual culture with 8 fungi (Fig. 1). The strong antagonistic activities (+++) against $B$. oryzae, $C$. echinochloae, $C$. orbiculare and $F$. oxysporum were observed in the dual culture plates, and the inhibition zones were 42.1, 30.9, 37.5, $31.6 \mathrm{~cm}^{2}$, respectively (Fig. 1 and Table 1). KT also showed moderate antagonistic activity (+ and ++ ) against other pathogenic fungi.

\subsection{Identification of Actinomycete Strain KT}

The actinomycete strain KT was isolated from a soil sample on the root of a tree collected at Wakayama, Japan. KT showed the filamentous growth and was gram-positive. It formed 2.0-3.0 mm white colony and aerial hyphae, but, did not form spores. The $16 \mathrm{~S}$ rDNA sequence of actinomycete strain KT shows high sequence similarity to genus Streptomyces, and the closest similarity with that of S. lavenduligriseus NRRL B-3173 ${ }^{\mathrm{T}}$ (Accession No. NR043824) with $99.6 \%$ similarity (Fig.2).

\subsection{Infection Control of Rhizoctonia Damping-off of Cucumber by Strain KT}

As shown in Fig.3B, symptom of $R$. solani infection was observed on the third day after inoculation in the control experiment, and severe damage was observed from days 6 to 8. But in the KT treated pots, only a weak infection was observed as shown in Figs. 3A, B. Since the clear suppression of $R$. solani growth was observed in cucumber which belongs to Cucurbitaceae, we performed the same assay using other plant species, qing-geng-cai in the family of Brassicaceae, in order to reveal whether the effect depends on host plants. The first infection was observed on the day 7 in the control experiment, and it was 4 days later compared with that of cucumber, but the severe damage was observed from days 15 to 20. However, in the KT treated experiment, strong suppressive effect against the infection by $R$. solani was clearly observed (Figs. 4A, B). Since the remarkable inhibitory effect by KT against the infection of $R$. solani was observed in the Hyponex medium, a sterilized and nutrition-rich medium, the similar experiment was done using the unsterilized soil, which is similar to the actual field. As shown in Figs. 5,6, KT showed a noticeable inhibitory effect against the infection by $R$. solani and protected cucumber and qing-geng-cai from the infection even in the non-sterile soil.

\subsection{Detached Leaf Assay of Isolated Actinomycete Strain KT against B. oryzae}

As the clear suppressive effect against $R$. solani was observed, another important phytopathogenic fungus $B$. oryzae, which causes disease symptoms mainly in high value field crops in the family Poaceae, including rice, maize, wheat, was used. To assess whether actinomycete KT could control the plant pathogen B. oryzae, A. sativa (oat) was used for detached leaf assay. Oat leaves were treated with or without KT. It was observed that leaves with soaking treatment of KT culture inhibited the growth of the plant pathogen and showed almost no lesion on the leaves, while the leaves of non-treated plants were covered by the pathogens as show in Fig.7A (top panels). The KT-treated leaves showed little mycelial formation of $B$. oryzae even on the final day of the test, and the disease severity was also low (Fig.7B). 


\section{Discussion}

There are a variety of microorganisms in the rhizosphere of plants. Interaction between plants and microorganisms influence on the plant growth in a positive or negative manner. Biological control agents are thought to be able to promote the growth of plants and suppress plant pathogens. Actinomycete is one of the potential biological control agents for plant disease and they are known to produce useful antimicrobial compounds.

Growth of 8 plant pathogens was inhibited in vitro assay by dual culture method, and strain KT had the strongest antifungal activity in the actinomycetes that were collected in our library. These results suggested the presence of antifungal metabolites which control wide range of plant pathogens. In this study, a novel strain Streptomyces sp. KT was evaluated as a biological control agent against cucumber damping-off, qing-geng-cai damping-off caused by $R$. solani and oat leaf blight caused by B. oryzae.

Disease suppressive potential of KT was tested by cucumber or qing-geng-cai infection control assay. The strain KT was able to protect the seedlings of cucumber or qing-geng-cai from $R$. solani. Infection control tests were performed on both Hyponex medium and unsterilized soil. Both tests showed successful suppression against plant pathogens. Previous studies often use culture medium and sterilized soil, but few reports use unsterilized soil. The experiments using unsterilized soil cannot avoid the influence of microorganisms present in the unsterilized soil, and thus, the focused microorganism is difficult to proliferate. However, KT could inhibit the growth of phytopathogenic fungi under the unsterilized soil condition. This result suggests that pretreatment with KT culture can reduce the infection of a plant pathogen even in the open fields.

In this study, we tried infection control tests on not only Rhizoctonia damping-off but also Brown spot (B. oryzae). B. oryzae is known to cause brown spot in the Poaceae family, and brown spot is diseases that cause important losses between 1-10\%. (Sarvary et al., 2000, 2006) Disease area of brown spot is the third largest in Japan, following blast rice blight (neck rot) and sheath blight (Matsumoto et al., 2016). Control of brown spot is an important issue for the rice cultivation area. KT can significantly delay the infection of $B$. oryzae in the detached leaf assay. Therefore, KT may be useful as a biological control agent to manage many plant pathogens in view of the results of dual culture assay.

The results of phylogenetic analysis based on 16S rDNA sequences indicate that actinomycete strain KT belongs to the cluster of the genus Streptomyces, however, the strain KT showed different phylogenetic position with $S$. lavenduligriseus NRRL B-3173 ${ }^{\mathrm{T}}$. Differences of 6 base in the 16S rDNA base sequence was observed between $S$. lavenduligriseus NRRL B-3173 ${ }^{\mathrm{T}}$ and strain KT. Among the difference of the segment, 2 bases are suggested to be caused by insertion/deletion, and the remaining 4 bases was clearly different. From these results, KT was identified as Streptomyces sp., which closely related to strain S. lavenduligriseus NRRL B-3173 ${ }^{\mathrm{T}}$. S. lavenduligriseus is known to produces pentenomycin and polyene macrolides (Jiayue et al., 2016). Pentenomycin is antibacterial compound and is not effective against fungi. On the other hand, polyene macrolides show a broad antifungal spectrum, and inhibition of plant pathogens has been reported, which may suggest that the antifungal compound of KT has also a similar structure (Kim et al., 2012). As far as we know, there is no report for biological control agents using S. lavenduligriseus. Therefore, research on the use of strain $\mathrm{KT}$ is considered to contribute to the development of novel biological control agents in the future.

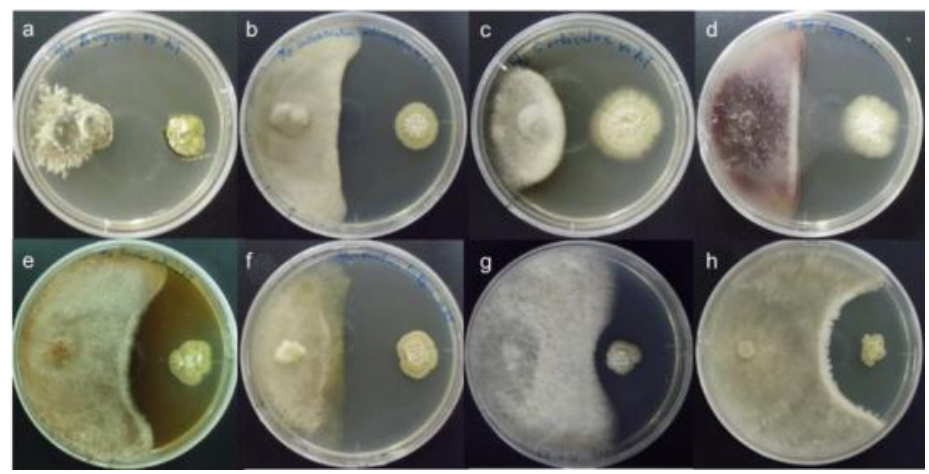

Figure 1. Dual culture assay of actinomycete strain KT (right) against plant pathogens (left). Strain KT and plant pathogens were inoculated on the PDA medium at the same time. (a) Bipolaris oryzae, (b) Colletotrichum echinochloae, (c) Colletotrichum orbiculare, (d) Fusarium oxysporum, (e) Fusarium solani, (f) Monilinia fructigena, (g) Phytophthora infestans, (h) Rhizoctonia solani 


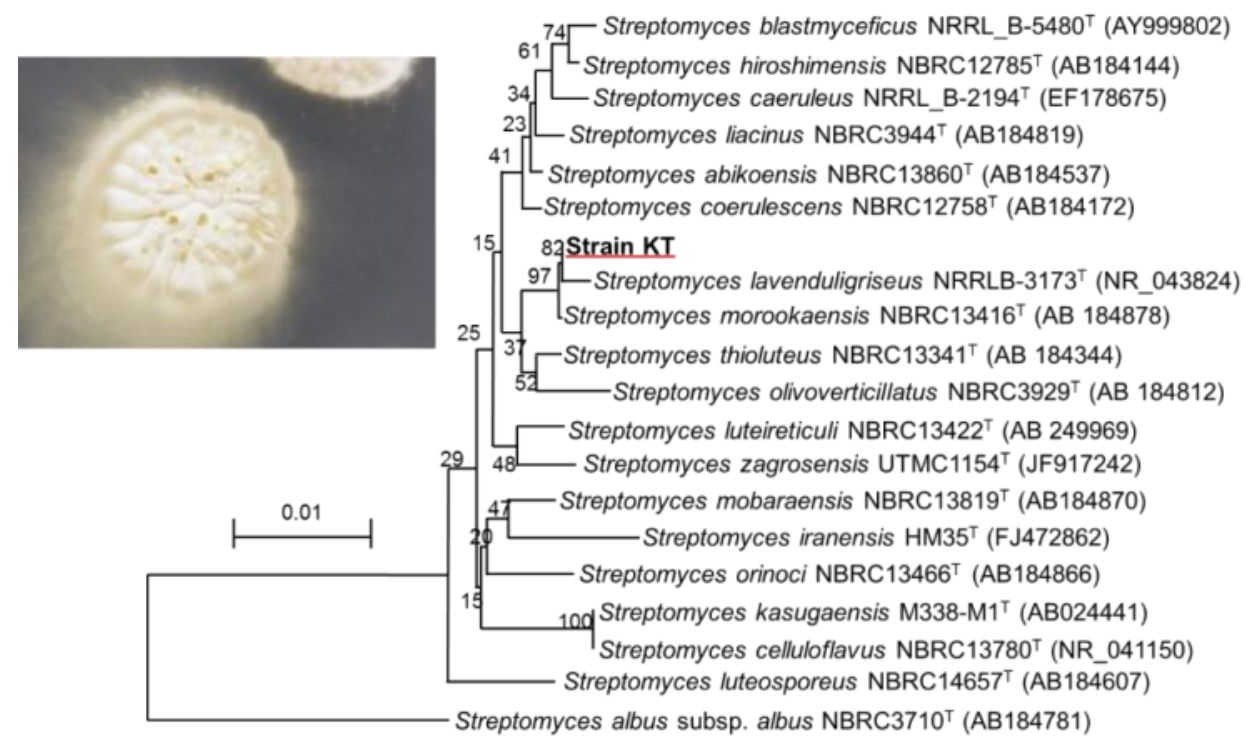

Figure 2. 16S rDNA gene sequences showing relationships between Streptomyces isolates and related Streptomyces type strain species. Numbers at nodes indicate levels of bootstrap support based on a neighbour-joining analysis of 1000 resampled datasets
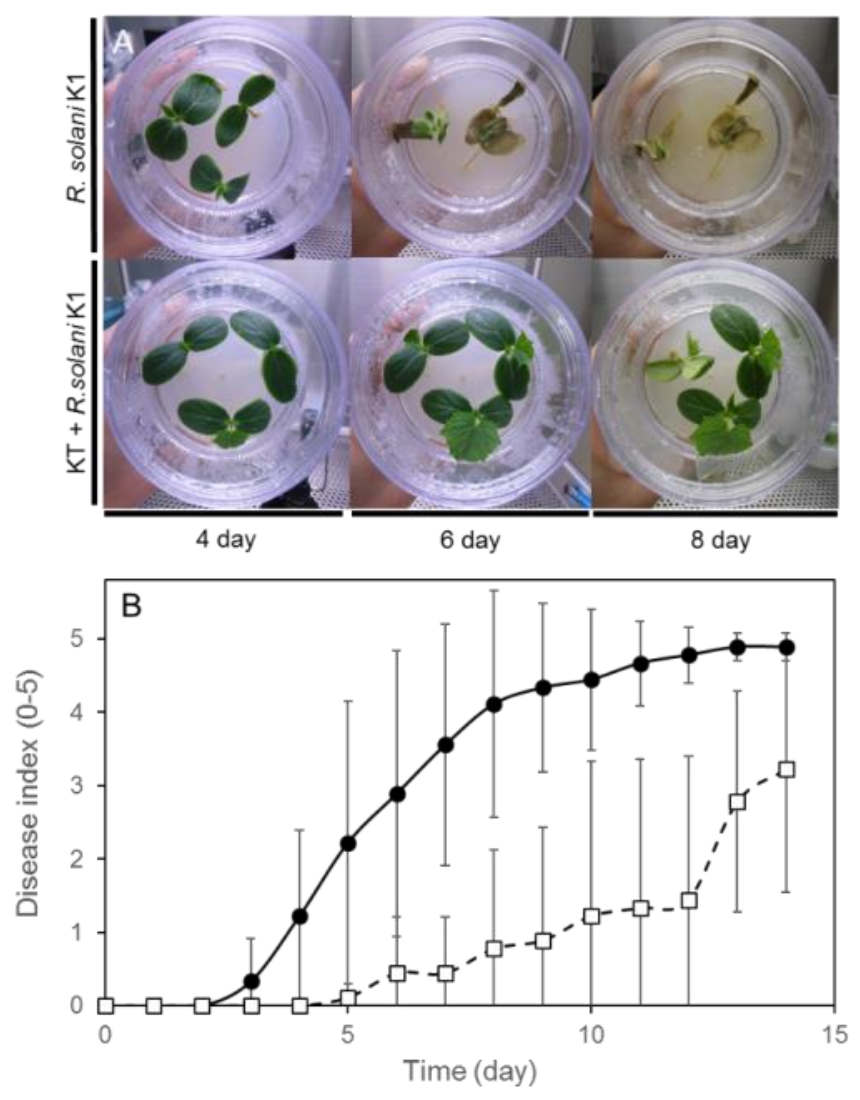

Figure 3. Infection control of strain KT against cucumber damping-off ( $R$. solani). The photograph of infection control of $R$. solani by strain KT(A). Reduction of disease severity of cucumber damping-off by strain KT(B). Inoculated with only $R$. solani (closed circle). Inoculated with $R$. solani and strain KT (open square). Disease severity index was developed based on the time period during the test that seedlings became infected and was calculated on a 0-to-5 value. The same experiment was repeated three times, and the average value and standard deviation were shown 

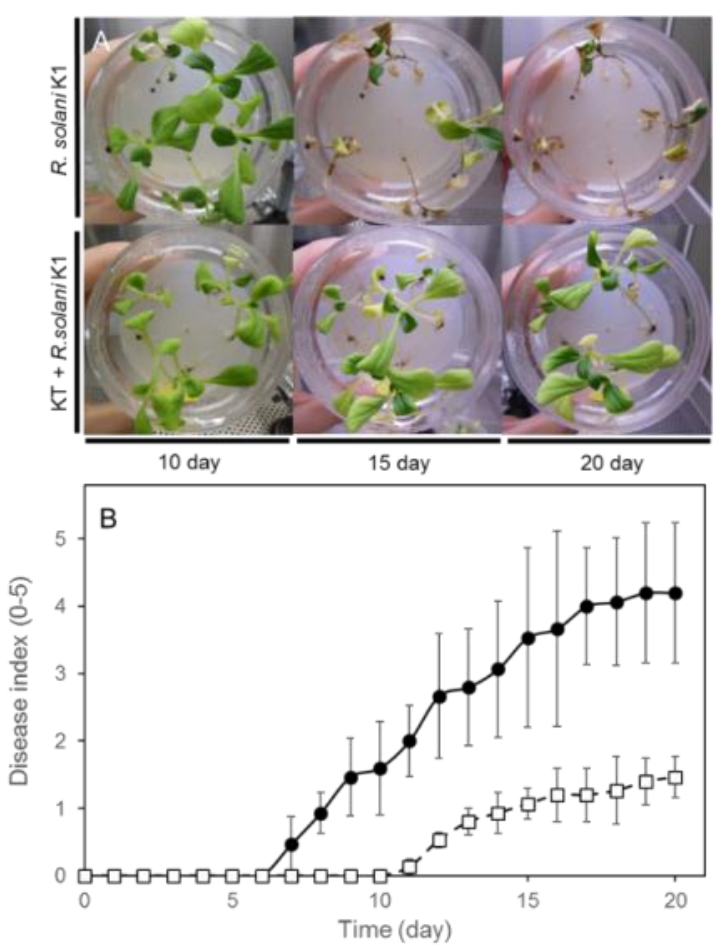

Figure 4. Infection control of strain KT against qing-geng-cai damping-off ( $R$. solani). The photograph of infection control of $R$. solani by strain KT(A). Reduction of disease severity of qing-geng-cai damping-off by strain KT (B). Inoculated with only $R$. solani (closed circle). Inoculated with $R$. solani and strain KT (open square). Disease severity index was developed based on the time period during the test that seedlings became infected and was calculated on a 0 -to-5 value. The same experiment was repeated three times, and the average value and standard deviation were shown
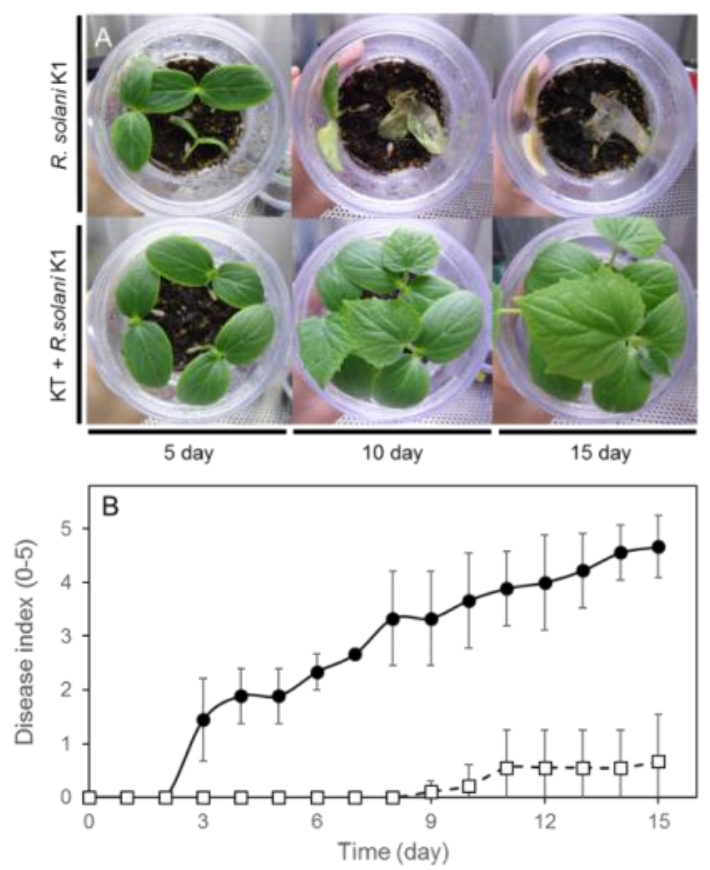

Figure 5. Infection control of strain KT against cucumber damping-off (R. solani) under unsterilized soil condition. The photograph of infection control of $R$. solani by strain KT (A). Reduction of disease severity of cucumber damping-off by strain KT (B). Inoculated with only $R$. solani (closed circle). Inoculated with $R$. solani and strain KT (open square). Disease severity index was developed based on the time period during the test that seedlings became infected and was calculated on a 0 -to- 5 value. The same experiment was repeated three times, and the average value and standard deviation were shown 

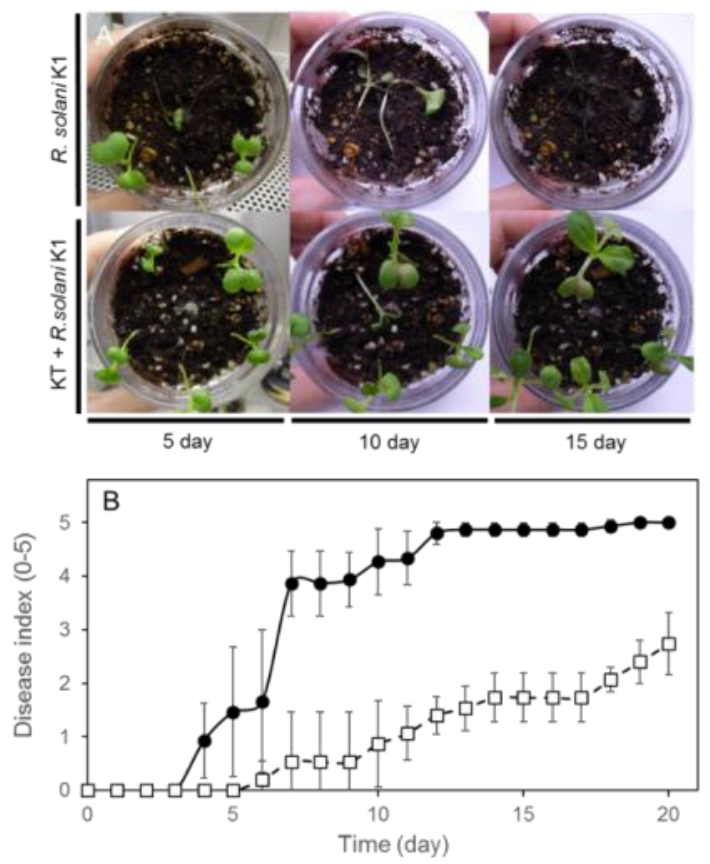

Figure 6. Infection control of strain KT against qing-geng-cai damping-off ( $R$. solani) under unsterilized soil condition. The photograph of infection control of $R$. solani by strain KT (A). Reduction of disease severity of qing-geng-cai damping-off by strain KT (B). Inoculated with only $R$. solani (closed circle). Inoculated with $R$. solani and strain KT (open square). Disease severity index was developed based on the time period during the test that seedlings became infected and was calculated on a 0 -to- 5 value. The same experiment was repeated three times, and the average value and standard deviation were shown
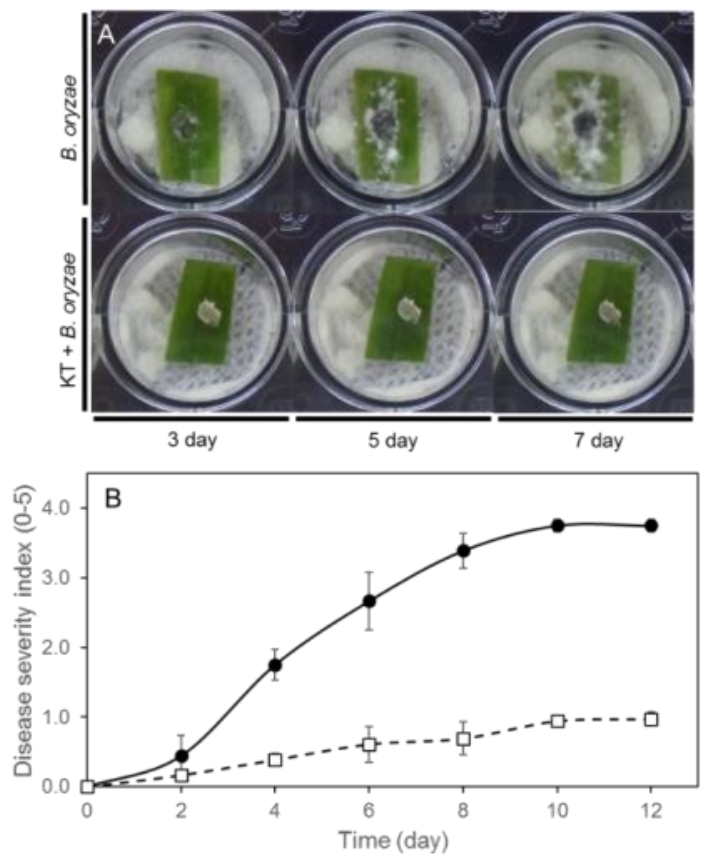

Figure 7. Detached leaf assay of strain KT against Bipolaris leaf blight. The top panels showed the result inoculated with $B$. oryzae and bottom panels showed that inoculated with $B$. oryzae and strain KT (A). Reduction of disease severity of Oat leaf blight (B. oryzae) by strain KT was clearly observed (B). Inoculated with only $B$. oryzae (closed circle). Inoculated with $B$. oryzae and strain KT (open square). Disease severity index was developed based on the time period during the test that seedlings became infected and was calculated on a 0 -to- 4 value (B). The same experiment was repeated three times, and the average value and standard deviation were shown 
Table 1. Antifungal activity of actinomycete strain KT against plant pathogens on PDA.

\begin{tabular}{llcc}
\hline Plant pathogens & Crops & Inhibition zone area $\left(\mathrm{cm}^{2}\right)$ & Inhibition index* \\
\hline Bipolaris oryzae & rice & $42.1 \pm 0.6$ & +++ \\
Colletotrichum echinochloae & Japanese millet & $30.9 \pm 0.5$ & +++ \\
Colletotrichum orbiculare & watermelon & $37.5 \pm 0.8$ & +++ \\
Fusarium oxysporum & cabbage & $31.6 \pm 0.5$ & +++ \\
Fusarium solani & rice & $18.6 \pm 0.1$ & + \\
Monilinia fructigena & apple & $29.6 \pm 0.5$ & ++ \\
Phytophthora infestans & tomato & $14.1 \pm 0.2$ & + \\
Rhizoctonia solani & lawn grass & $11.7 \pm 0.2$ & + \\
\hline
\end{tabular}

(*inhibition zone,$+<20 \mathrm{~cm}^{2} ;++, 20-30 \mathrm{~cm}^{2} ;+++,>30 \mathrm{~cm}^{2}$ )

\section{References}

Agricultural Development Economics Division Food and Agriculture Organization of the United Nations, World agriculture towards 2030/2050: the 2012 revision (2012). http://www.fao.org/home/en/

Al-Sa'di, M. A., Drenth A., Deadman, M. L., De Cock, A. W. A. M., \& Aitken, E. A. B., (2007). Molecular characterization and pathogenicity of Pythium species associated with damping-off in greenhouse cucumber (Cucumis sativus) in Oman. Plant pathology, 56, 140-149. https://doi.org/10.1111/j.1365-3059.2006.01501.x

Asaka, O., \& Shoda, M. (1996). Biocontrol of Rhizoctonia solani Damping-Off of Tomato with Bacillus subtilis RB14 Applied and Environmental Microbiology, 62(11), 4081-4085. https://www.ncbi.nlm.nih.gov/pmc/articles/PMC1388978/

Banville, J. G. (1989). Yield losses and damage to potato plants caused by Rhizoctonia solani Kuhn. American Potato Journal, 66(12), 821-834. https://doi.org/10.1007/BF02853963

Castillo, M., B., Dunn, F., M., Navarro, G., K., Meléndez, H., F., Ortiz, H., M., Guevara, E., S., \& Palacios, H., G. (2016). Antifungal performance of extracellular chitinases and culture supernatants of Streptomyces galilaeus CFFSUR-B12 against Mycosphaerella fijiensis Morelet. World Journal of Microbiology and Biotechnology, 32-44. https://doi.org/10.1007/s11274-015-1993-0

Clevo, W., \& Clem, T. (2001). Why farmers continue to use pesticides despite environmental, health and sustainability costs. Ecological Economics, 39(3), 449-462. https://doi.org/10.1016/S0921-8009(01)00238-5

Department of Economic and Social and Economic Affairs, World Population Prospects: The 2017 Revision United Nations, (2017). http://www.un.org/en/development/desa/population/index.shtml

Jiayue, Y., Zhijun, Y., Yu, Y., Min, R., Yongheng, L., \& Mei, G. (2016). Three novel polyene macrolides isolated from cultures of Streptomyces lavenduligriseus. The Journal of Antibiotics, 69, 62-65. http://dx.doi.org/doi:10.1038/ja.2015.76

Kim, D., J., Han, W., J., Hwang, C., I., Lee, D., \& Kim, S. B. (2012). Identification and biocontrol efficacy of Streptomyces miharaensis producing filipin III against Fusarium wilt. Journal of Basic Microbiology, 52, 150-159. http://dx.doi.org/10.1002/jobm.201100134

Lee, F. N., \& Rush, C. M., (1983). Rice Sheath Blight: A Major Rice Disease. Plant Disease, 67(7), 829-832. https://doi.org/10.1094/PD-67-829.

Lewis, A. J., \& Papavizas, C. G., (1980). Integrated control of Rhizoctonia fruit rot of cucumber. Phytopathology Journal, 70(2), 85-89. https://doi.org/10.1094/Phyto-70-85.

Lewis, A. J., \& Lumsden, D. R., (2001). Biocontrol of damping-off of greenhouse-grown crops caused by Rhizoctonia solani with a formulation of Trichoderma spp. Crop Protection, 20(1), 49-56. https://doi.org/10.1016/S0261-2194(00)00052-1

Matsumoto, K., Sato, H., Ota, C., Seta, S., Yamakawa, T., Suzuki, H., \& Nakayama, Y. (2016). A new method for evaluating field resistance to brown spot in rice. Breeding Research, 18(3), 103-111.

http://doi.org/10.1270/jsbbr.18.103

Marchetti, A. M., \& Bollich, C. N., (1991). Quantification of the relationship between sheath blight severity and 
yield loss in rice. Plant Disease, 75(8), 773-775 https://doi.org/ 10.1094/PD-75-0773

Ogoshi, A. (1987). Ecology and Pathogenicity of Anastomosis and Intraspecific Groups of Rhizoctonia Solani Kuhn. Annual Review of Phytopathology, 25, 125-143. https://doi.org/10.1146/annurev.py.25.090187.001013

Parry, W. D. (1990). Diseases of potato. In: Plant Pathology in Agriculture. Cambridge University Press, Cambridge, UK.

Sakdapetsiri, C., Fukuta, Y., Aramsirirujiwet, Y., Shirasaka, N., \& Kitpreechavanich, V. (2016). Antagonistic activity of endo- $\beta-1,3$-glucanase from a novel isolate, Streptomyces sp. 9X166, against black rot in orchids. Journal of Basic Microbiology, 56(5), 469-479. https://doi.org/10.1002/jobm.201500709

Sanglier, J., Haag, H., Huck, T., \& Fehr, T. (1996). Section Review; Anti-infectives: Review of actinomycetes compounds 1990-1995. Expert Opinion on Investigational Drugs, 5(2), http://dx.doi.org/10.1517/13543784.5.2.207

Savary, S., Willocquet, L., Elazegui, F. A., Castilla, N. P., \& Teng, P. S., (2000). Rice pest constraints in tropical Asia: quantification of yield losses due to rice pests in a range of production situations, Plant Disease, 84(3), 357-369. https://doi.org/10.1094/PDIS.2000.84.3.357

Savary, S., Teng, S., P., Willpcquet, L., \& Nutter, F., W. (2006). Quantification and modeling of crop losses: A review of purposes. Annual Review of Phytopathology, 44, 89-112.

https://doi.org/10.1146/annurev.phyto.44.070505.143342

Ushiyama, K., Nishimura, J., \& Aono, N. (1987) Dampping-off of feather cockscomb (Celosia argentea L. var. cristata O. Kuntze) caused by Rhizoctonia solani Kuhn. Kanagawa Prefecture Horticultural Experiment Station Research Report, 34, 33-37.

\section{Copyrights}

Copyright for this article is retained by the author(s), with first publication rights granted to the journal.

This is an open-access article distributed under the terms and conditions of the Creative Commons Attribution license (http://creativecommons.org/licenses/by/4.0/). 